\title{
ASPECTOS CLÍNICOS E RADIOGRÁFICOS DA COLUNA CERVICAL DE BEZERROS SUBMETIDOS À PROVA DO LAÇO
}

\author{
Raquel Mincarelli Albernaz, ${ }^{1}$ Deborah Penteado Martins Dias, ${ }^{1}$ Marco Augusto Giannocaro da Silva, ${ }^{2}$ \\ Lina Maria Wehrle Gomide, ${ }^{1}$ Julio Carlos Canola ${ }^{3}$ e José Corrêa Lacerda Neto ${ }^{3}$
}

1. Pós-graduandas, doutorado, Faculdade de Ciências Agrárias e Veterinárias, UNESP, Jaboticabal, SP. -

E-mail: raquel_albernaz@hotmail.com

2. Professor adjunto I, Universidade Federal do Tocantins. Câmpus Araguaína

3. Professores adjuntos, Departamento de Clínica e Cirurgia Veterinária. Faculdade de Ciências Agrárias e Veterinárias, UNESP, Jaboticabal, SP.

\section{RESUMO}

A modalidade de rodeio denominada prova do laço de bezerro tem sido questionada por causa da ocorrência de lesões nas vértebras cervicais ocasionadas pela tração da corda no pescoço dos bezerros. Neste trabalho, avaliaram-se quinze bezerros mestiços, machos ou fêmeas, entre cinco e seis meses de idade submetidos experimentalmente à prova do laço. Os animais foram laçados três vezes por semana, em dias alternados, durante cinco semanas, somando o total geral de 225 laçadas. Avaliaram-se os bezerros mediante exame clínico geral e neurológico ao início da primeira, durante a terceira e ao término da quinta semana experimental.
Radiografias simples e contrastadas das vértebras cervicais foram efetuadas ao início da primeira e ao término da quinta semana de experimento. Acompanharam-se os métodos de laçadas por médico veterinário, sendo classificados qualitativamente em fortes ou fracos. Não se encontraram alterações clínicas e radiográficas nos animais durante o experimento. $\mathrm{O}$ rigor da laçada foi considerado forte em $77 \%$ dos casos. Nas condições deste trabalho, a prova do laço não causou lesão na região cervical, embora tenha sido considerado um procedimento agressivo e rude com o bezerro.

PALAVRAS-CHAVES: Bezerro, mielografia, neurologia clínica, traumatismo.

\section{ABSTRACT}

\section{CLINICAL AND RADIOGRAPHIC ASPECTS OF CERVICAL COLUMN IN CALVES SUBMITTED TO CALF ROPING}

The modality of roundup calf roping has been questioned on the occurrence of possible injuries to the cervical vertebrae caused by the rope tension in the calf's neck. In this work, 15 calves, male or female, ages varying from five to six months experimentally submitted to calf roping were evaluated. The procedure was carried out three times per week, in alternated days, during five weeks, adding the total of 225 lassoes. The calves had been evaluated by means of general and neurological clinical examination at the beginning of the first week, during the third one and at the end of the fifth experimental week. Simple and contrasted x-rays of

KEY WORDS: Calves, myelography, clinical neurology, trauma. the cervical vertebrae had been made at the beginning of the first one and at the end of the fifth week of experiment. The lassoes methods had been observed and classified qualitatively in weak or strong. Clinical and radiographic alterations in the animals during the experiment had not been found. The severity of the lassoes was considered strong in $77 \%$ of the cases. The fact that clinical and radiographic abnormalities have not been found indicates that the occurrence of cervical injuries in calves submitted to calf roping is not as high as divulged, however, it was considered an aggressive and rude procedure. 


\section{INTRODUÇÃO}

A modalidade de rodeio denominada prova do laço de bezerro tem sido criticada por causa das condições de conforto ambiental e psíquico oferecidas ao bezerro, assim como pelas possíveis lesões provocadas pela tração da corda sobre o pescoço dos animais.

O exame físico pode levantar suspeitas a respeito da localização da fratura com base na observação e na palpação do desvio dorsoventral ou lateral dos processos vertebrais dorsais espinhais (REBHUN, 2000). Os sinais de fratura vertebral com compressão medular são agudos e geralmente não progressivos, com exceção das fraturas não deslocadas que, subsequentemente, se deslocam comprimindo a medula espinhal (REBHUN, 2000; MARQUES et al., 2004).

Lesões medulares severas na região cervical (C1-C5) estão associadas ao comprometimento da movimentação dos membros torácicos e pélvicos, sendo os sinais clínicos caracterizados por tetraplegia espástica e ataxia. As compressões medulares nesse local provocam sinais mais intensos em membros pélvicos, em virtude do posicionamento mais superficial de seus tratos motores quando comparados aos membros torácicos (STÕBER, 1987; MILLS et al., 1988; BRAUND et al., 1990; BORGES et al., 1997; BORGES et al., 2000). As compressões leves podem acometer apenas os membros pélvicos (STÕBER, 1987; MILLS et al., 1988; BRAUND et al., 1990; BORGES et al., 1997; BORGES et al., 2000). A perda total da sensação dolorosa dificilmente é encontrada, pois um trauma de grande magnitude na região de $\mathrm{C} 1$ a $\mathrm{C} 5$ leva à falência respiratória com consequente óbito do animal (STÕBER, 1987; BRAUND et al., 1990; LE COUTEUR \& CHILD, 1995). Em caso de compressões severas, se pode ter ainda o acometimento e disfunção da bexiga (MILLS et al., 1988).

Determinadas lesões na região cranial da medula espinhal torácica podem provocar a síndrome de Horner (ptose da pálpebra superior, miose e protusão da terceira pálpebra acompanhada geralmente por sudorese unilateral da região facial). Esses sinais ocorrem em razão da lesão dos nervos simpáticos no tronco vagossimpático localizados anatomicamente na medula espinhal torácica cranial até próximo à órbita (BORGES et al., 2000; BORGES, 2004).
As fraturas cervicais podem envolver qualquer parte da vértebra, porém o corpo vertebral, processo transverso e espinhoso são os locais mais comumente afetados. O diagnóstico radiográfico dever ser feito com base na observação do alinhamento da coluna cervical, tamanho, forma e radiopacidade vertebral (DOUGLAS \& WILLIAM, 1970; MORGAN, 1972; WALKER, 1998).

A delimitação da medula espinhal com auxílio de meios de contraste positivo (mielografia) no bezerro é tecnicamente viável, porém só é praticada em casos especiais, em virtude do alto custo do material e conhecimento técnico que reserva (STÕBER, 1987).

Em que pese o fato de haver inúmeras informações não científicas sugerindo a ocorrência de lesões no pescoço de bezerros utilizados em prova de laço, não há, até o presente momento, informações científicas seguras que comprovem este fato. Diante dessas circunstâncias, o objetivo deste trabalho foi avaliar possível lesão traumática cervical decorrente da prática da prova do laço de bezerro, mediante a realização de exame clínico geral e específico da região cervical, além de utilização de técnicas radiográficas simples e contrastadas.

\section{MATERIAL E MÉTODOS}

Foram utilizados quinze bezerros mestiços, machos ou fêmeas, entre cinco e seis meses de idade, pesando entre 90 a $120 \mathrm{~kg}$, provenientes de quatro criatórios na região de Jaboticabal, SP.

Durante o período do experimento, permaneceram alojados em centro de treinamento hípico particular, onde foram desverminados (Ivermectina $-0,2 \mathrm{mg}$ / $\mathrm{Kg}$ ), vacinados para Clostridium perfringens $B / C / D$, Clostridium septicum, Clostridium nouyi B, Clostidium sordellii e Clostridium chavoei (Sintoxan polivalente), marcados e numerados a ferro incandescente.

Os animais foram submetidos à prova experimental do laço, realizada de maneira semelhante à prova oficial dessa modalidade de rodeio, três vezes por semana, em dias alternados, durante cinco semanas consecutivas.

Avaliaram-se os bezerros por meio de exame clínico geral e neurológico ao início da primeira, durante a terceira e ao término da quinta semana experimental. O exame clínico geral foi realizado pela avaliação do 
peso corpóreo em quilogramas, altura de cernelha em centímetros e do escore corporal (RADOSTITS, 2000). Efetuaram-se aferição dos parâmetros vitais (frequência cardíaca, respiratória e temperatura retal), inspeção da cútis, linfonodos, mucosas, veia jugular e exame do sistema músculo esquelético. O exame neurológico incluiu avaliações do comportamento e postura corporal, avaliação da marcha e sinais de nervos cranianos.

Procedeu-se às projeções radiográficas com os animais contidos em decúbito lateral esquerdo sobre um colchão. Padronizaram-se $56 \mathrm{kVp}$ ou $58 \mathrm{kVp}, 20 \mathrm{~mA}$ e $0,06 \mathrm{seg}$ para as radiografias simples e $58 \mathrm{kVp}, 20 \mathrm{~mA}$ e 0,06 seg para radiografias contrastadas com distância de 76,2 centímetros do chassi.

Submeteram-se aleatoriamente cinco animais do grupo experimental para ser realizada mielografia antes do início e ao término do período experimental. Os animais foram previamente sedados utilizando xilazina $2 \%$, na dose $0,05 \mathrm{mg} / \mathrm{kg}$ por via intramuscular e após dez minutos administraram-se $2 \mathrm{mg} / \mathrm{kg}$ de cetamina $5 \%$ por via intravenosa. Após tricotomia e antissepsia da região da articulação atlanto occipital, a cisterna magna foi puncionada com agulha estéril 40x12 até atingir o espaço subaracnoide. Colheram-se e desprezaram-se aproximadamente $10 \mathrm{~mL}$ de líquido cefalorraquidiano. $\mathrm{O}$ agente iodado não iônico, iopamidol (Iopamiron ${ }^{\circledR} 370$ - Berlimed), na dose $0,25 \mathrm{~mL} /$ $\mathrm{kg}$, foi injetado lentamente no espaço epidural e a seguir realizadas as projeções radiográficas previamente descritas.

Procedeu-se às provas experimentais de forma semelhante à prova oficial, seguindo as regras da Confederação Nacional de Rodeio (CNAR, 2006) pelo mesmo cavaleiro profissional da modalidade. Os métodos de laçadas foram acompanhados e classificados qualitativamente em fortes ou fracos

Para amostra pareada na escala nominal, escore corporal, empregou-se o teste de McNemar. Para amostras pareadas na escala quantitativa, peso e altura de cernelha, foi usado o teste T pareado. Para análise das amostras frequência cardíaca, frequência respiratória, frequência rumenal e temperatura em graus Celsius (FC, FR, FRUM e $\mathrm{T}^{\circ} \mathrm{C}$ respectivamente), utilizou-se análise de variância de medidas repetidas e, nos casos em que houve diferença significativa, efetuou-se o teste de Tukey a 5\% (ZAR, 1999).

\section{RESULTADOS E DISCUSSÃO}

Os valores de peso e altura de cernelha variaram significativamente entre o início da primeira semana (T1) e o término da quinta semana de experimento (T3) (Tabela 1).

TABELA 1. Média \pm desvio-padrão (DP) para os valores de peso e altura de cernelha dos bezerros ao início da primeira semana (T1) e ao término da quinta semana de experimento (T3)

\begin{tabular}{lccc}
\hline Variável & T1 & T3 & $\begin{array}{c}\text { Valor de P no } \\
\text { teste } \\
\text { t-student* }\end{array}$ \\
\hline Peso $(\mathrm{kg})$ & $104,60 \pm 14.64$ & $109,47 \pm 17,24$ & 0.032 \\
Altura $(\mathrm{cm})$ & $104,14 \pm 13.65$ & $106,07 \pm 12,98$ & 0.005 \\
\hline * P $>0,05$ não significativo a 5\%; $<<0,05$ significativo a $5 \% ; \mathrm{P}<0,01$ \\
significativo a 1\%.
\end{tabular}

Os únicos critérios adotados na escolha dos animais referem-se ao fato de serem mestiços e pesarem, no início do experimento, entre 90 e $120 \mathrm{~kg}$, respeitando as regras oficiais da modalidade, as quais determinam que animais com peso corpóreo acima de $140 \mathrm{~kg}$ não devem ser utilizados. Esse aspecto, de relativa importância durante o treinamento, limita a utilização dos animais a um período de aproximadamente dois ou três meses, além do qual estes estão muito leves ou muito pesados para a prática da modalidade.

O escore corporal se manteve entre bom e moderado na escala de RADOSTITS (2000), e não houve alteração significativa entre o início da primeira semana e o término da quinta semana de experimento, de acordo com o Teste de McNemar $(\mathrm{p}=0,3173)$.

Neste experimento, a avaliação da auscultação de cavidades torácica e abdominal não revelou anormalidade, tanto na qualidade quanto na frequência de sons produzidos, durante o período experimental. Ao exame da cútis, dos linfonodos, das mucosas e da veia jugular, não se encontraram alterações nos parâmetros avaliados. O TPC avaliado nas mucosas orais não pigmentadas foi dois segundos durante todo o período experimental.

A análise neuromotora não revelou alteração nos parâmetros estudados durante o período experimental. Não se registraram quaisquer anormalidades nos mo- 
vimentos de levantar-se e caminhar. Os músculos e os processos espinhosos cervicais não apresentaram, durante inspeção e palpação, aumentos de volume ou sensibilidade dolorosa local. Após o procedimento da laçada, os animais permaneceram em piquete para pastoreio e todos os animais foram capazes de flexionar o pescoço, apreender e deglutir alimentos. MARQUES et al. (2004) relataram evidências de dor cervical caracterizada por rigidez do pescoço e orelhas, dificuldade de movimentação da cabeça a despeito da manutenção do apetite em caso de compressão medular cervical em bezerro.

No presente estudo, não houve qualquer evidência de anormalidade da marcha caracterizando quadro de ataxia, hipertermia, espasticidade ou paresia, o que levou a descartar a ocorrência de lesões da medula espinhal, durante o período de observação.

WATSON et al. (1985) e DOIGE et al. (1990) relataram caso de compressão medular cervical de origem congênita em bezerro associada à incapacidade de se manter em posição quadrupedal, embora continuassem alertas e responsivos ao meio ambiente.

WHITE et al. (1978) referiram a ocorrência de subluxação atlanto axial e compressão medular em cinco bezerros jovens com sinais clínicos caracterizados por tetraparesia, ataxia, estado mental alerta e responsivos ao meio externo.

Sinais progressivos de dificuldade em manter a posição quadrupedal e ocorrência de ataxia, paresia e posteriormente tetraparesia foram associados à presença de abscesso na região cervical comprimindo a medula espinhal (SHERMAN \& AMES, 1986). Este relato se diferenciou dos mencionados anteriormente, uma vez que há evolução progressiva dos sinais clínicos, ao passo que nos casos de mal formação ou lesão traumática o quadro é agudo e estável.

Neste experimento não ocorreu qualquer alteração dos pares de nervos cranianos estudados. Já BRAUN et al. (2003), em relato de compressão medular cervical, descreveram reflexo de ameaça diminuído ou ausente e ptose palpebral em bovinos adultos.

Tais resultados podem ser justificados pelo fato de este experimento ter sido realizado sob condições especiais, embora não controladas. O laçador responsável pela execução dos procedimentos é um cavaleiro experiente, envolvido profissionalmente com a prova do laço de bezerro há sete anos. Essa experiência lhe possibilita, em alguns casos, suavizar manualmente o impacto da laçada, uma vez que este gesto diminui, em tese, a ocorrência de derrube do bezerro em decúbito lateral ou ventral. Sob o ponto de vista das regras da modalidade, o fato de o bezerro não ser derrubado é vantajoso, uma vez que encurta o tempo de duração da prova. No entanto, existem outros fatores como aceleração e ângulo de incidência que interagem na produção das forças exercidas sobre o pescoço, os quais são totalmente incontroláveis.

A análise radiográfica da região cervical em projeção lateral deste experimento revelou, ao início da primeira semana e ao término da quinta semana experimental, desalinhamento entre as vértebras $\mathrm{C} 3 \mathrm{e}$ $\mathrm{C} 4 \mathrm{em}$ todos os animais. Em seis animais, o desalinhamento ocorreu também entre as vértebras $\mathrm{C} 2$ e $\mathrm{C} 3$ e em cinco animais entre $\mathrm{C} 4$ e $\mathrm{C} 5$. O exame radiográfico contrastado demonstrou que esses desalinhamentos vertebrais não produziram compressão em medula (Figura 1).

Tal fato pode estar relacionado à largura do canal vertebral do bovino, aparentemente maior que a apresentada por outras espécies de mamíferos domésticos. Segundo DYCE et al. (1990), os ruminantes apresentam um canal medular relativamente largo na altura do atlas.

Nos casos em estudo, atribuiu-se o desalinhamento entre as vértebras cervicais como sendo uma característica anatômica de bezerro, uma vez que os sinais clínicos observados nos exames realizados nos indivíduos antes do início do experimento (controle) não diferiram daqueles registrados ao término do período experimental.

Somando o total de 225 laçadas, $17,77 \%$ foram classificadas como sem aproveitamento técnico (SAT), ou seja, o cavaleiro não conseguiu completar a prova por erro da laçada. Considerando apenas as laçadas efetuadas com êxito, $77 \%$ foram consideradas como uma forte tração no pescoço dos animais e 34\% levaram o animal ao solo, por causa da força exercida pela corda.

Diante dessas considerações, não se descarta a possibilidade de ocorrência de lesão quando o bezerro é laçado por pessoas menos experientes, as quais não possuem habilidade suficiente para controlar a intensidade da força do laço sobre as estruturas do pescoço. 

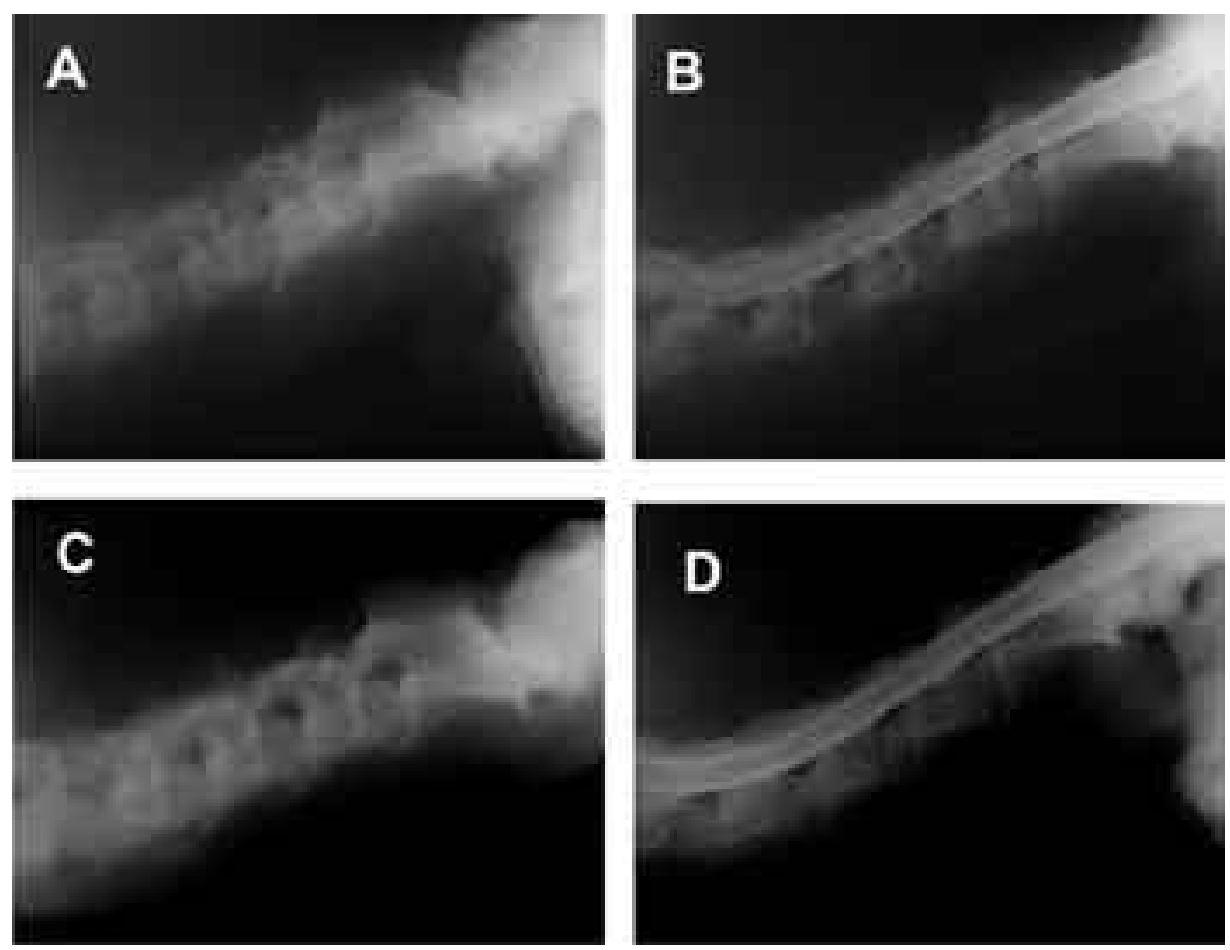

FIGURA 1. Avaliações radiográficas simples (A) e contrastada (B) da região cervical ao início experimento e ao término do período experimental (C) e (D). Notar desalinhamento entre corpos vertebrais 3 e 4.

\section{CONCLUSÕES}

A modalidade de rodeio denominada prova do laço de bezerro não causou, sob as condições experimentais pré-estabelecidas, alteração nos parâmetros clínicos e radiográficos avaliados neste trabalho.

O desalinhamento encontrado entre os corpos das vértebras cervicais durante exame radiográfico não provocou alteração nos parâmetros estudados e é provavelmente devido à característica anatômica dos animais.

Experimentos adicionais envolvendo laçadores em diferentes níveis de capacitação para o esporte precisam ser realizados para que se possa reunir maior número de informações, considerando que até o presente momento não existe relato literário envolvendo este aspecto da modalidade de rodeio estudada.

\section{COMITÊ DE ÉTICA}

Protocolo n ${ }^{\circ}$ 002508-04 - CEBEA - Comissão de Ética e Bem-Estar Animal - FCAV, Unesp, Jaboticabal.

\section{REFERÊNCIAS}

BORGES, A. S. Exame clínico geral ou de rotina. In: FEITOSA, F. L. F. Semiologia veterinária: a arte do diagnóstico. São Paulo: Roca, 2004. p. 507-526.

BORGES, A. S.; MENDES, L. C. N.; GRAFKUCHEMBUCK, M. R. Exame neurológico em grandes animais. Parte II - Medula espinhal: eqüino com incoordenação motora. Revista de Educação Continuada, v. 3, n. 2, p. 3-15, 2000.

BORGES, A. S.; SAPATERA, A. C.; MENDES, L. C. N. Avaliação dos reflexos espinhais em bezerros. Ciência Rural, v. 27, n. 4, p. 613-617, 1997.

BRAUN, U.; FLUCKIGER, M.; GERSPACH, C.; GREST, P. Clinical and radiographic findings in six cattle with cervical diskospondylitis. Veterinary Record, v. 153, n. 20, p. 630-632, 2003.

BRAUND, K. G.; SHORES, A; BRAWNER, W. R. The etiopatology, pathology and pathophysiology of an acute spinal cord trauma. Veterinary Medicine, p. 684-721, 1990.

DOIGE, C. E.; TOWNSEND, E. D.; JANSEN, E. D.; MCGOWWAN, M. Congenital spinal stenosis in beef calves in western Canada. Veterinary Pathology, v. 27, p. 16-25, 1990. 
DOUGLAS, S. W.; WILLIAM, H. D. The vertebral column and rib cage. In: Veterinary radiological interpretation.

Philadelphia: Lea \& Febiger, 1970. p. 72-75.

DYCE, K. M.; SACK, W. O.; WENSING, C. J. G. O pescoço, o dorso e a cauda dos ruminantes. In: Tratado de anatomia veterinária. Rio de Janeiro: Guanabara Koogan, 1990. p. 427431.

MARQUES, L. C.; CADIOLI, F. A. NETTO, A. C.; ÁVILA, L. G.; CANOLA, J. C.; ALESSI, A. C. Abscessos em coluna vertebral de bezerros e cordeiros: aspectos neurológicos. Revista de Educação Continuada, v. 7, p. 15-22, 2004.

MILLS, L. L.; DAHLSTROM, C.; HOGAN, P. M. Recognizing cervical spinal cord compression in young calves. Veterinary Medicine, p. 1181-1185, 1988.

MORGAN, J. P. Vertebral column. In: Radiology in veterinary orthopedics. Philadelphia: Lea \& Febiger, 1972. p. 223-225.

RADOSTITS, O. M. Exame clínico de bovinos adultos e bezerros. In: RADOSTITS, O. M.; MAYHEW, I. G. J.; HOUSTON, D. M. Exame clínico e diagnóstico em veterinária. Rio de Janeiro: Guanabara Koogan, 2000. p. 118-139.
REBHUM, W. C. Doenças neurológicas. In: Doenças do gado leiteiro. São Paulo: Roca, 2000. p. 501-544.

SHERMAN, D. M.; AMES, T. R. Vertebral body abscesses in cattle: a review of five cases. Journal of American Veterinarian Medical Associaton, v. 188, n. 6, p. 608-611, 1986.

STÕBER, M. Sistema nervoso central. In: ROSENBERGER. Exame clínico dos bovinos. Rio de Janeiro: Guanabara Koogan, 1987. p. 341-362.

WALKER, M. A. The vertebrae. In: THRALL, D. E. Textbook of veterinary diagnostic radiology. 3. ed. Philadelphia: W. B. Saunders, 1998. p. 81-88.

WATSON, A. G.; WILSON, J. H.; COOLEY, A. J. Occipitoatlanto-axial subluxation in an ataxic calf. Journal of American Veterinary Medical Association, v. 187. n. 7, p. 740-742, 1985.

WHITE, M. E.; PENNOCK, P. W.; SEILER, R. J. Atlanto-axial subluxation in five young cattle. Canadian Veterinary Journal, v. 19, p. 79-82, 1978.

ZAR, J. H. Biostatistical analysis. New Jersey: Prince Hall, 1999. 